\title{
Evaluation of TWO METHOdS OF PREDiCTING MLC LEAF POSITIONS USING EPID MEASUREMENTS
}

Laure Parent, Joao Seco ${ }^{\dagger}$, Phil M Evans

5 Joint Department of Physics, The Institute of Cancer Research and The Royal Marsden NHS Foundation Trust, Downs Road, Sutton, SM2 5PT, United Kingdom

David R Dance

Joint Department of Physics, The Institute of Cancer Research and The Royal

10 Marsden NHS Foundation Trust, Fulham Road, London, SW3 6JJ, United Kingdom

Andrew Fielding

School of Physical and Chemical Sciences, Queensland University of Technology, 15 Q337 Gardens Point Campus, Brisbane, Queensland 4001, Australia

${ }^{\dagger}$ Present address:

Department of Radiation Oncology, Francis Burr Proton Therapy Center, Massachusetts General Hospital, Harvard medical school, Boston, USA

\section{ABSTRACT}

In intensity modulated radiation treatments (IMRT), the position of the field edges and the modulation within the beam are often achieved with a multileaf collimator (MLC). During the MLC calibration process, due to the finite accuracy of leaf 25 position measurements, a systematic error may be introduced to leaf positions. Thereafter leaf positions of the MLC depend on the systematic error introduced on each leaf during MLC calibration and on the accuracy of the leaf position control system (random errors). This study presents and evaluates two methods to predict the systematic errors on the leaf positions introduced during the MLC calibration. The two presented methods are based on a series of EPID measurements. A comparison with film measurements showed that the EPID could be used to measure leaf positions without introducing any bias. The first method, referred as the "central leaf method", is based on the method currently used at this centre for MLC leaf calibration. It mimics the manner in which leaf calibration parameters are specified in 
35 the MLC control system and consequently is also used by other centres. The second method, a new method proposed by the authors and referred as the "individual leaf method", involves the measurement of two positions for each leaf $(-5$ and $+15 \mathrm{~cm})$ and the interpolation and extrapolation from these two points to any other given position. The central leaf method and the individual leaf method predicted leaf 40 positions at prescribed positions of $-11,0,5$ and $10 \mathrm{~cm}$ within $2.3 \mathrm{~mm}$ and $1.0 \mathrm{~mm}$ respectively, with a standard deviation (SD) of 0.3 and $0.2 \mathrm{~mm}$ respectively. The individual leaf method provided a better prediction of the leaf positions than the central leaf method. Reproducibility tests for leaf positions of -5 and $+15 \mathrm{~cm}$ were performed. The reproducibility was within $0.4 \mathrm{~mm}$ on the same day and $0.4 \mathrm{~mm}$ six

45 weeks later (1 SD). Measurements at gantry angles of 0,90 and $270^{\circ}$ for leaf positions of -5 and $+15 \mathrm{~cm}$ showed no significant effect of gravity. The individual leaf method could be used in various applications to improve the accuracy of radiotherapy treatment from planning to delivery. Three cases are discussed: IMRT beam verification, MLC calibration and dose calculation. 


\section{INTRODUCTION}

In intensity modulated radiation therapy (IMRT), modulation in the beam is often achieved with a multileaf collimator (MLC). During the MLC calibration process, due to the finite accuracy of leaf position measurements, a systematic error may be introduced to leaf positions. Moreover, a $2.0 \mathrm{~mm}$ tolerance exists on the leaf positions ${ }^{1}$, leading to a possible range of $\pm 2.0 \mathrm{~mm}$ for a given position. Figure 1 shows leaf positions measured at the end of the MLC calibration process for one of

60 the banks of a linear accelerator. It can be seen that an error on leaf positions is still present but it is accepted as within tolerances. Thereafter leaf positions of the MLC depend on the systematic error introduced on each leaf during MLC calibration and on the accuracy of the leaf position control system (random errors). Variation between the planned and actual leaf positions could introduce a significant difference in the

65 magnitude and position of the dose delivered. A recent paper has highlighted the impact of the MLC leaf positioning accuracy on IMRT plan verification results ${ }^{2}$. In this study, the authors irradiated an ion chamber with IMRT segments. They repeated the same measurements but moved the leaves randomly in between. They observed variations up to $13 \%$ on ion chamber measurements when the MLC leaves partially 70 covered the ion chamber.

The purpose of this study was to develop a leaf position prediction method, taking into account the inaccuracies in leaf positions introduced by the MLC calibration. Two methods based on a series of EPID measurements were developed and tested. The central leaf method was based on the method currently used at this centre for

75 MLC leaf calibration. It mimicked the manner in which leaf calibration parameters are specified in the MLC control system and consequently is also used by other centres. The individual leaf method, a new method proposed by the authors, consisted 
in measuring the leaf positions at two points for each individual leaf and interpolating between these two points. The reproducibility of leaf positions was tested in the short and long term (same day and six weeks apart, respectively), and as a function of gantry angle.

A method predicting MLC leaf positions could be used for various applications and three cases will be discussed: IMRT beam verification, MLC calibration and dose calculation.

85

\section{MATERIALS AND METHODS}

\section{A- Materials}

\section{1- Linac}

The measurements were performed on a Precise linac (Elekta Ltd, Crawley, UK), operating at $6 \mathrm{MV}$. The crossplane and inplane directions are defined respectively by the Y-and the X-axes (IEC 60601-2-1 convention $^{3}$ ). The convention for the axes used in this document is shown on the first diagram of figure 2. Precise linacs are equipped with a 40 leaf pair MLC. Each leaf has a width of $1 \mathrm{~cm}$ at the isocentre and travels parallel to the $\mathrm{Y}$-axis. The leaf edge is rounded in the $\mathrm{Y}$ direction and stepped

95 in the X-direction. The leaf positions are monitored continually by optical imaging, with a CCD camera, of reflectors located at the upper surface of each leaf ${ }^{4}$.

For Elekta linacs, the standard 40 leaf MLC calibration ${ }^{5}$ consists of placing the central leaf pair (leaf pair 20) at two calibration points $(-5$ and $+15 \mathrm{~cm}$ at the isocentre) and fitting a straight line calibration between them. The other leaves are then calibrated by applying an offset correction. The offset is determined by measuring the position difference between leaf 20 and leaf $i(1 \leq i \leq 40)$ of a given bank while both banks are positioned at $7.5 \mathrm{~cm}$. The tolerance on leaf 20 position and 
on the position of the other leaves compared to leaf 20 is $1.0 \mathrm{~mm}$. By comparing the leaf positions to leaf pair 20, the uncertainty and hence the tolerance on leaf position measurement is doubled to $2.0 \mathrm{~mm}$ for all leaves but leaf pair 20. The leaf positions are usually measured with film during the MLC calibration.

\section{2- EPID}

Leaf positions were measured on portal images, produced with an iViewGT system

110 (Elekta Ltd, Crawley, UK) equipped with an amorphous silicon flat panel detector (PerkinElmer Inc, Santa Clara, USA). The active area of the detector is $41 \times 41 \mathrm{~cm}^{2}$ and is located at $160 \mathrm{~cm}$ from the source. There are $1024 \times 1024$ pixels in the image. The physical pixel size of the detector is $0.4 \mathrm{~mm}$, projecting to $0.25 \mathrm{~mm}$ at the isocentre.

115 Images acquired with the EPID were corrected for bad pixels and calibrated as indicated in equation 1, where DFI stands for dark field image, an image acquired in the absence of any radiation to correct for electronic noise, and FFI stands for flood field image, an image acquired with a field covering the entire area of the detector to correct the gain for each individual pixel.

120 corrected_image $(x, y)=\frac{\text { raw_image }_{-}(x, y)-\operatorname{DFI}(x, y)}{\operatorname{FFI}(x, y)-\operatorname{DFI}(x, y)}$

\section{B- Definitions}

The following terminology is used:

- The position of a leaf in the MLC specified by the user is referred as the prescribed position.

- The actual leaf position, as measured by the EPID, is referred as the true position. It is subject to the finite accuracy of the calibration process. 
- The leaf position predicted with the model is referred as the predicted position.

130

\section{C- Leaf position prediction models}

Two methods to predict the position of a given leaf were tested.

The central leaf method: This method is based on the method currently used at this centre for MLC leaf calibration. It mimics the Elekta MLC calibration procedure: for each bank, the true position of leaf 20 was measured for prescribed positions of -5

135 and $+15 \mathrm{~cm}$. The values were then used to determine $\mathrm{a}$ and $\mathrm{b}$ in equation 2 .

True_position(leaf 20) $=a^{*}$ Prescribed_position $($ leaf 20) $+b$

An offset for every other leaf was determined, compared to leaf 20 position, at a position of $+5 \mathrm{~cm}$, halfway between the two calibration points used for leaf 20 . In practice, Elekta uses a position of $+7.5 \mathrm{~cm}$ to measure this offset. The predicted

140 position of any leaf was then determined by equation 3 , with a and $\mathrm{b}$ obtained from equation 2.

Predicted_position $($ leaf $i)=a^{*}$ Prescribed_position $($ leaf $i)+b+$ offset $($ leaf $i)$

The individual leaf method: For each leaf, the true positions at -5 and $+15 \mathrm{~cm}$ were measured and used to determine a and $\mathrm{b}$ in equation 2 . The predicted position was then determined by equation 4 .

Predicted_position $($ leaf $i)=a($ leaf $i) *$ Prescribed_position $($ leaf $i)+b($ leaf $i)$

Each method was then tested by predicting the true positions for prescribed positions of $-11,0,5$, and $10 \mathrm{~cm}$ for each leaf and by comparing them with the true positions. 


\section{D- Image acquisition and analysis}

\section{1- Image acquisition}

In order to test the two methods of leaf position prediction, images were acquired with the leaf banks paired as follow (Y2 bank/Y1bank): -11/20, -5/15, 0/10, 5/5, 10/0, 15/-5 and 20/-11 (figure 2) to minimise the number of acquisitions. The EPID sensitive area is located at approximately $160 \mathrm{~cm}$ from the source and cannot be moved vertically. As the area of detection is $41 \times 41 \mathrm{~cm}^{2}$, it was only possible to image 24 leaves at a time. Two images were therefore necessary to image all leaves in a bank for a given position: the first covered leaves 1 to 24 (top acquisition) and the second covered leaves 17 to 40 (bottom acquisition). When results are presented for the whole leaf banks, leaf 1-20 and 21-40 positions were extracted respectively from

165 the top and bottom acquisitions. The EPID was translated laterally to image different sets of leaf pairs.

\section{2- Image analysis}

A central $6 \times 6 \mathrm{~cm}^{2}$ field was used to determine the position of the central axes.

170 This field size was chosen such that it was small enough to fit in the EPID for all EPID positions but large enough to have a flat profile at the centre of the field. Central crossplane and inplane profiles of this field were plotted and normalised to the signal at the centre. The origin of the image was defined as the middle position between the two $50 \%$ points of each plot. The $50 \%$ points were determined by

175 interpolation between the two nearest pixel values. The central axes were then defined as the vertical and horizontal lines intersecting at the origin of the image. 
It is necessary to measure, and correct if applicable, the collimator rotation. As there is a tolerance of $0.5^{\circ}$ on the collimator angle, it can introduce an error of up to 2 $\mathrm{mm}$ on the position of the most external leaves and for large leaf offsets. The rotation was determined by measuring the distance from the horizontal central axis to the $\mathrm{X}$ jaws at -10 and $+10 \mathrm{~cm}$ on $y$-axis on $15 /-5$ and $-5 / 15$ fields (figure 3 ). When a gantry rotation was applied, the collimator rotation correction was recalculated. The correction applied ranged between 0 and $0.5^{\circ}$ for all measurements. This method assumes that the leaf travel direction is exactly parallel to the edge of the $\mathrm{X}$ jaws. In practice, in our institution, the $\mathrm{X}$ jaws are calibrated against the MLC, using films placed on treatment couch at the isocentre. The horizontality of the couch was verified with a clock gauge. The maximum distance between the jaws and a given leaf should not vary by more than $1.0 \mathrm{~mm}$ over the whole length of the collimation system (40 $\mathrm{cm})$. This corresponds to a tilt of $0.14^{\circ}$ and defines the accuracy limit of the method.

Baker et $a l^{6}$ reported a tilt of the EPID in the inplane direction, resulting in a magnification in the inplane direction on one side of the EPID being slightly different from the other side of the EPID. In the present work, the EPID tilt was measured on an EPID image of a $22 \times 22 \mathrm{~cm}^{2}$ field at the isocentre (figure 4) in which the field limits were defined by the jaws only (the leaves were retracted by $1 \mathrm{~cm}$ behind the 195 jaws). Crossplane profiles were plotted at $X=+10 \mathrm{~cm}$ and $X=-10 \mathrm{~cm}$ at the isocentre and normalised to the maximum in the profile. The EPID tilt $\alpha$ was obtained according to equation 5, where Field_width $(+10 \mathrm{~cm})$ and Field_width $(-10 \mathrm{~cm})$ denote the distance between the two $50 \%$ points of the profiles at $X=+10 \mathrm{~cm}$ and $X=-10 \mathrm{~cm}$ respectively and $d$, the distance between the two profiles.

$$
\sin \alpha=\frac{\left(\frac{\text { Field_width }(-10 \mathrm{~cm})}{\text { Field_width }(+10 \mathrm{~cm})}-1\right) * 100}{d}
$$


This method allows measurement of the EPID tilt for any gantry angle with a precision of $0.2^{\circ}$. A correction of the images for the EPID tilt was applied by scaling the leaf positions according to their position in the image. The position of the tilt axis was chosen to give the closest match between the film and EPID measurements.

205 The leaf edge positions were extracted from the images by plotting profiles across the leaf pairs. The profiles were normalised to the maximum in the profile of leaf pair 20. Leaf edges were measured at the $50 \%$ of the profile by interpolation between the two nearest points. The origin of the coordinates system used for the measurements was at the centre of a $6 \times 6 \mathrm{~cm}^{2}$ field and central axes were defined as the vertical and

210 horizontal lines intersecting at this point after the rotation correction was applied (as defined previously).

\section{3- Film comparison}

In radiotherapy, the plane of reference is at the isocentre and the positions of the

215 leaves are defined relative to this plane. If one wants to use the EPID to measure the leaf positions, it is necessary to be able to relate the EPID measurements to this plane of reference. By comparing leaf positions measured with films placed at the isocentre, it is possible to verify that the tilt and rotation corrections applied allow us to obtain the correct leaf positions at the isocentre. A comparison of the leaf positions measured 220 with film and EPID for 15/-5 and -5/15 fields were performed. The films were placed at a depth of $5 \mathrm{~cm}$ in solid water at the isocentre on the couch. The absence of couch tilt was verified with a clock gauge. For both detectors, two images were acquired in order to cover the entire MLC bank length (leaf 1-24 and leaf 17-40). The leaf positions were therefore measured twice for 8 pairs of leaves (leaf pairs 17-24), which

225 provided on estimate of the standard error of the measurements. 


\section{E- Reproducibility of leaf positions}

The reproducibility of leaf positions was tested in the short term (same day) and the long term (6 weeks apart).

The short term reproducibility was assessed by repeating the same measurement (5/15 field) 10 times while changing the MLC settings to arbitrary fields between each measurement. These fields were chosen such that the leaves were either outside or inside the beam. As the acquisition had to be performed in two steps to cover the entire MLC, the same arbitrary fields were used in the same order for the second set of measurements.

235 To assess the long term reproducibility of the method, $-5 / 15$ and $15 /-5$ field EPID measurements (figure 2) were repeated six weeks later and compared with the predicted positions based on the previous measurements.

The effect of gravity was assessed by repeating $-5 / 15$ and 15/-5 fields at gantry angles of $90^{\circ}$ and $270^{\circ}$.

\section{RESULTS}

\section{A- Film comparison}

An EPID tilt of $0.4,-0.3$ and $0^{\circ}$ in the $\mathrm{X}$ direction and $0,0.2$ and $0^{\circ}$ in the $\mathrm{Y}$

245 direction (IEC 60601-2-1 convention ${ }^{3}$ ) for gantry angles of 0,90 and $270^{\circ}$ was found. For the $0^{\circ}$ gantry angle, this measurement was checked with a clock gauge and found to be within the uncertainties of measurements. No tilt variation was observed when the EPID was moved horizontally.

The mean standard errors of measurements averaged across leaf pairs 17-24 for 250 the film, the EPID without any tilt correction and the EPID with a tilt correction were 
respectively $0.4,0.4$ and $0.3 \mathrm{~mm}$. The accuracy of the measurements with both films and the EPID is $0.1 \mathrm{~mm}$. Figure 5 presents the difference between EPID and film measurements for two different positions of the leaf banks $(-5$ and $+15 \mathrm{~cm})$ and with and without the correction for the EPID tilt. The root mean square error of the EPID measurements compared with the film measurements was $0.6 \mathrm{~mm}$ without a tilt correction applied to the EPID images and $0.5 \mathrm{~mm}$ with a tilt correction applied to the EPID images. The tilt correction improved the agreement of the EPID measurements with the films on average, but not significantly.

These results confirmed that the EPID can be used to measure the leaf positions at 260 the isocentre and in the rest of this paper, all the leaf positions were measured with the EPID only and with the EPID tilt correction applied.

\section{B- Leaf position prediction methods}

Figure 6 summarises the results obtained with the two leaf position prediction

265 methods. Figures $6 a$ and $6 \mathrm{~b}$ present the difference between the true and the prescribed position for prescribed positions of $-11,-5,0,5,10$ and $15 \mathrm{~cm}$. All the values, apart for leaf pair 1, lie within the $2.0 \mathrm{~mm}$ tolerance on the leaf positions due to the methodology used for the MLC calibration, as described earlier. Figures 6c and $6 \mathrm{~d}$ present the difference between the predicted position using the central leaf method

270 and the true position for prescribed positions of $-11,-5,0,5,10$ and $15 \mathrm{~cm}$. The central leaf method failed to predict accurately enough the leaf position, as differences between the predicted and true positions of up to $2.3 \mathrm{~mm}$ were observed. The largest differences were observed for the outermost leaves. Figures 6e and $6 f$ present the difference between the predicted and the true position using the individual leaf 275 method for prescribed positions of $-11,-5,0,5,10$ and $15 \mathrm{~cm}$. The individual leaf 
method predicted the leaf position to within $1.0 \mathrm{~mm}$. For the $-11,0,5,10 \mathrm{~cm}$ data set, the absolute difference averaged across the leaves was $0.3 \mathrm{~mm}$ and $0.2 \mathrm{~mm}$ for the central and individual leaf method respectively and the standard deviation of the absolute difference was 0.4 and $0.2 \mathrm{~mm}$ for the central and individual leaf method respectively.

\section{C- Reproducibility of leaf positions}

For the reproducibility test on the same day, the maximum positional difference for a given leaf was $1.0 \mathrm{~mm}$ and the average maximum difference was $0.1 \mathrm{~mm}$ at a prescribed position of -5 or $+15 \mathrm{~cm}$. For a given leaf, the maximum standard deviation was $0.4 \mathrm{~mm}$ (leaves 1 to 4 ) and the average standard deviation was $0.2 \mathrm{~mm}$.

The results of the reproducibility test performed at 6 weeks are presented in figures $7 \mathrm{a}$ and $7 \mathrm{~b}$. All the leaf positions were reproduced to within $1.1 \mathrm{~mm}$ (Average= $0.0 \mathrm{~mm}, \mathrm{SD}=0.4 \mathrm{~mm}$ ) for a prescribed position of -5 or $+15 \mathrm{~cm}$.

The effect of gravity is presented in figure $7 \mathrm{c}$ and $7 \mathrm{~d}$ for a prescribed position of 5 or $+15 \mathrm{~cm}$. All the positions were reproduced to within $1.5 \mathrm{~mm}$. For gantry angles of $90^{\circ}$ and $270^{\circ}$, the average differences across the leaves were $-0.2 \mathrm{~mm}$ and $-0.2 \mathrm{~mm}$ and the standard deviations were $0.5 \mathrm{~mm}$ and $0.4 \mathrm{~mm}$ respectively. This difference may have been introduced by the finite accuracy in determination of the position of the origin of the image $(0.1 \mathrm{~mm})$ at each gantry angle. It is not a result of the effect of gravity as one would expect to observe the shift in different directions at 90 and $270^{\circ}$ gantry angles. Indeed the effect on the MLC, when the gantry is rotated, should be minimal as the optical control system of the leaf positions is stable with gantry position ${ }^{1}$. 


\section{DISCUSSION}

In this study, two methods to predict the true leaf positions of an MLC were developed and tested.

A comparison with film measurements showed that the EPID could be used to

305 measure leaf positions. An EPID tilt correction, as suggested by Baker et $a l^{6}$, was implemented. It improved the agreement of the EPID measurements with the films on average, but not significantly. A comparison of the true leaf position with the prescribed position (figures $6 \mathrm{a}$ and $6 \mathrm{~b}$ ) for prescribed positions of $-11,-5,0,5,10$ and $15 \mathrm{~cm}$ showed that differences up to $2.0 \mathrm{~mm}$ were observed and confirmed the need of 310 a prediction method of the exact leaf position.

Two methods were tested to predict the leaf position: the central leaf method mimicked the manner in which leaf calibration parameters are specified in the MLC control system and the individual leaf method, a new method proposed by the authors, involved the measurement of two positions for each leaf $(-5$ and $+15 \mathrm{~cm})$ and the interpolation and extrapolation between these two points for any other given position. The individual leaf method predicted the position with greater accuracy than central leaf method. As the same series of measurements was used to extract the coefficients and to test the prediction method, the next step was to test how reproducible the method was. For prescribed positions of -5 and $+15 \mathrm{~cm}$, the standard deviation of the long term reproducibility test $(\mathrm{SD}=0.4 \mathrm{~mm})$ was comparable to the short term reproducibility test $(\mathrm{SD}=0.4 \mathrm{~mm})$. The long term reproducibility test combines the effect of the inaccuracy of the positioning (short term test) and the drift in positioning with time. No significant variation of the standard deviation was observed between the two tests. Hence it can be concluded that no significant drift occurred during 6 
weeks. The frequency recommended by the Institute of Physics and Engineering in Medicine (IPEM) for the check of individual leaf positions is 6 months $^{7}$.

For all the reproducibility measurements, differences were larger for the outermost leaves. No satisfactory explanation to this was found and the question of the origin of these differences remains open.

It might be argued that a better prediction of the leaf positions would be obtained using more calibration points $(-10,-5,0,5,10,15 \mathrm{~cm}$ for example). However the average absolute difference between the predicted and the true position was $0.2 \mathrm{~mm}$ with the individual leaf method. The standard deviation of the short term reproducibility test being $0.4 \mathrm{~mm}$, it is unlikely that increasing the number of 335 calibration points will significantly improve the prediction.

The results presented in this study are specific to Elekta MLC. Other manufacturers, such as Varian and Siemens, use a different technology to monitor leaf positions and to calibrate their MLC $^{8}$. Both Varian and Siemens MLC leaf positions are controlled by encoders. The Varian MLC calibrates the leaf positions for one 340 position using an optical beam and an alignment jig $^{9}$. For the Siemens MLC, the calibration is performed at 4 positions $(-10,0,10$ and $20 \mathrm{~cm})$ using the light field and millimetre graph paper ${ }^{10}$. The technology being different, it is difficult to draw any conclusion regarding the application of this work for other manufacturers' MLC.

The first effect of the inaccuracy of a leaf position that could be expected is a 345 variation of the dose within the field, especially for small fields, as the output factor varies more rapidly for smaller fields. A recent paper $^{11}$ on narrow MLC defined fields showed that for field widths between 1 and $3 \mathrm{~cm}$, a variation of the field width by more than $0.50 \mathrm{~cm}$ is necessary before observing a significant variation $(>2 \%)$ of the dose. Such values are unlikely to happen for leaf position errors and an accuracy 
350 of the leaf position better than $2.0 \mathrm{~mm}$ is not necessary in terms of beam intensity. Although the results presented by Lydon $^{11}$ were obtained on a Varian linac (6MV photon beam), similar results are expected on the Elekta linac, as a similar photon energy was used in our experiments. The main impact of the leaf position accuracy will then be the determination of the position of the field edges.

A leaf position prediction method, such as the individual leaf method, could be used for various applications. Three cases can be considered: IMRT beam verification, MLC calibration and dose calculation. IMRT beam verification consists of predicting an image of an IMRT beam (reference image) and comparing it with a measured image (using films or EPID for example). By using the proposed leaf position prediction method to generate the reference image when verifying a beam, the discrepancies between the reference and the measured image due to the systematic errors resulting from the MLC calibration could be removed. But it would not have an impact on the accuracy of the delivered plan. However the methodology proposed in this paper could easily be used during the MLC calibration. Providing that a smaller tolerance on leaf positions is set during the MLC calibration, the accuracy of the delivered plan would increase and the systematic errors between the reference and the measured image resulting from the MLC calibration would decrease. From the series of tests conducted in this study, an accuracy on leaf positions of $1.0 \mathrm{~mm}$ is achievable. Ultimately the residual error on leaf positions after the MLC calibration could be incorporated in the treatment planning system using the individual leaf method, thus allowing the incorporation of the residual errors on leaf positions directly in the planning process. 
CONCLUSION

This study presented and evaluated two methods to determine the true leaf position of an MLC in order to account for the systematic errors introduced during MLC calibration. The best method (the individual leaf method) successfully predicted the leaf position to within $1.0 \mathrm{~mm}$. Although a leaf position error is not expected to affect segment intensity, it is still crucial to predict accurately the field edges. Three possible applications of leaf position prediction method were discussed: IMRT beam verification, MLC calibration and dose calculation, showing a great potential of the method to improve the accuracy of radiotherapy treatment from planning to delivery.

\section{AKNOWLEDGEMENTS}

The authors are grateful to Cancer Research UK and the Institute of Cancer Research for supporting this project under the programme reference SP2313/0201. They would also like to thank Elekta Ltd and PerkinElmer Inc for providing the data for the Monte Carlo modelling and useful comments on the manuscript. The help with the experiments from the staff of The Royal Marsden NHS Foundation Trust was much appreciated, especially from Mike Partridge for help with the development of the EPID image acquisition system, and from Ellen Donovan for help with the film measurements and analysis. 


\section{FIGURES}

Figure 1: Leaf positions measured at the isocentre with film after the MLC calibration for Y1 bank. Each leaf is represented by a bar. A position of $7.5 \mathrm{~cm}$ was prescribed to the leaves (bold 400 line). The maximum accepted deviation from the prescribed position is represented by dotted line. All leaves are within the $2.0 \mathrm{~mm}$ tolerance.

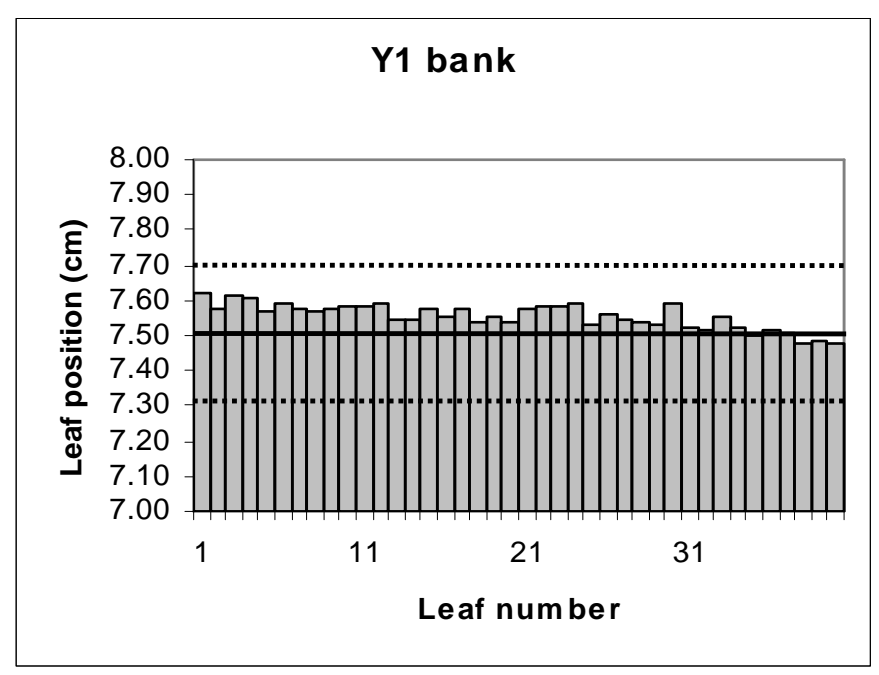


Figure 2: Fields used to measure the leaf positions (beam eye view). Central axes are represented by the black arrows. The $\mathrm{Y}$-jaws are represented in grey. The position of the leaf banks (Y2/Y1) is specified below the figures. The $\mathrm{X}$-jaws are not shown but were placed so that they minimised the irradiation outside the EPID.

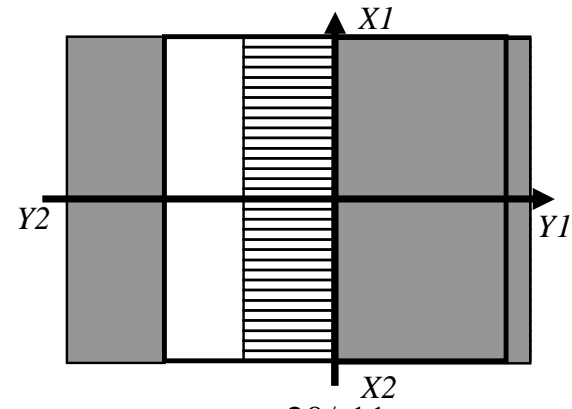

$20 /-11$

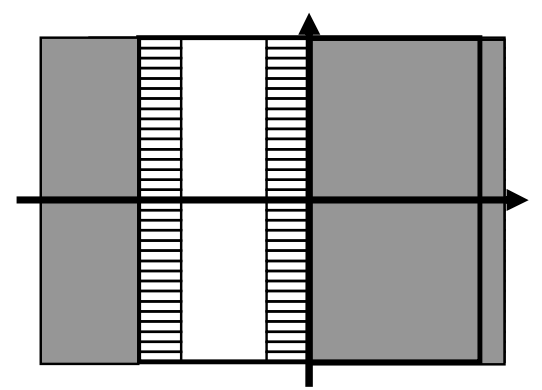

$15 /-5$

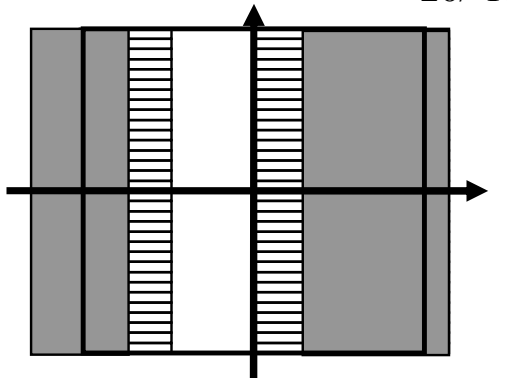

$10 / 0$

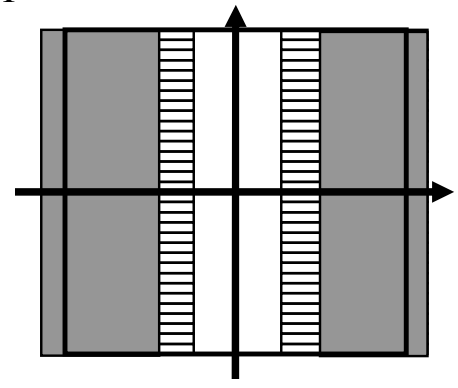

$5 / 5$

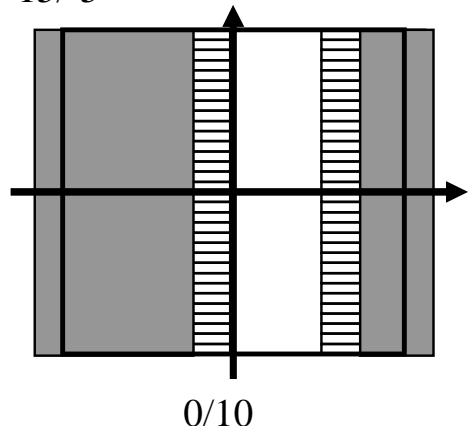

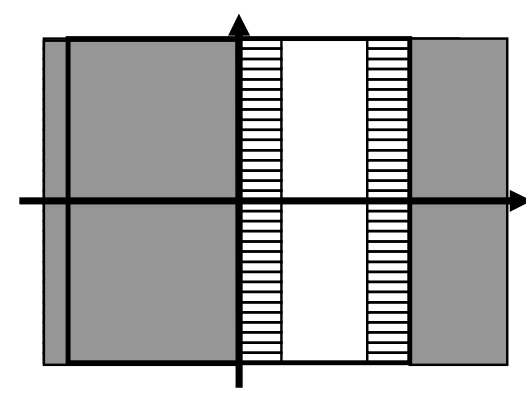

$-5 / 15$

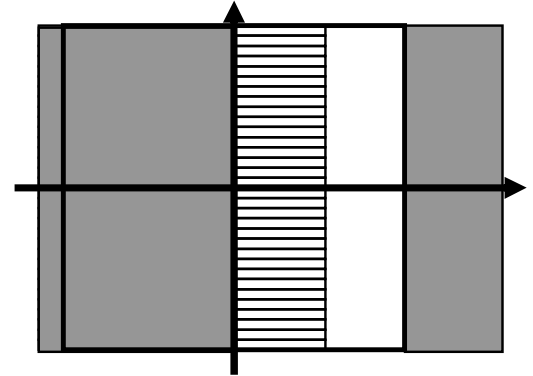

$-11 / 20$ 
Figure 3: The four EPID images used to determine the collimator rotation. The solid black squares represent the central pixel, as determined with the $6 \times 6$ field. The dotted lines are the central axes, if no collimator rotation is assumed. a and b represent the EPID images of 15/-5

field (leaves 1-24 and 17-40 respectively). $c$ and $d$ represent the EPID images of $-5 / 15$ field (leaves 1-24 and 17-40 respectively). The images were rotated until $d_{1}$ equalled $d_{3}$ and $d_{2}$ equalled $d_{4}$.
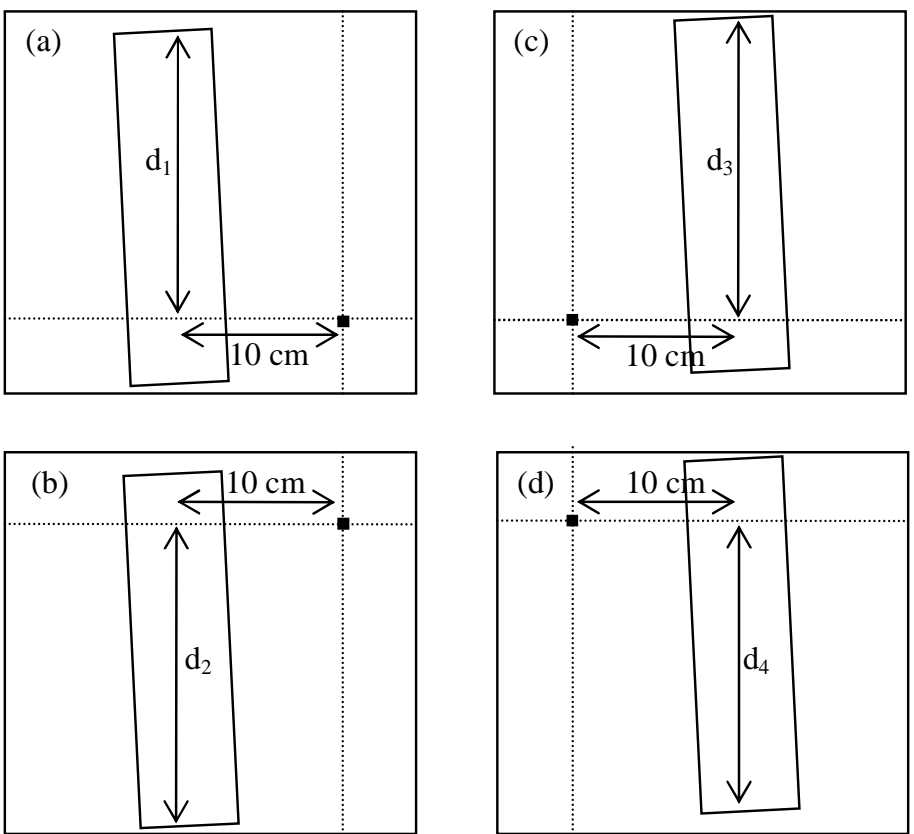
430 Figure 4: EPID tilt measurement. On an EPID image of a $22 \times 22 \mathrm{~cm}^{2}$ at the isocentre (represented by a thick line), the field width is measured at $X= \pm 10 \mathrm{~cm}$. The leaves were retracted by $1 \mathrm{~cm}$ behind the $Y$ jaws. Central axes are represented in dotted lines and $d$ represents the distance between the two measurements of the field width. The tilt is obtained according to equation 5.

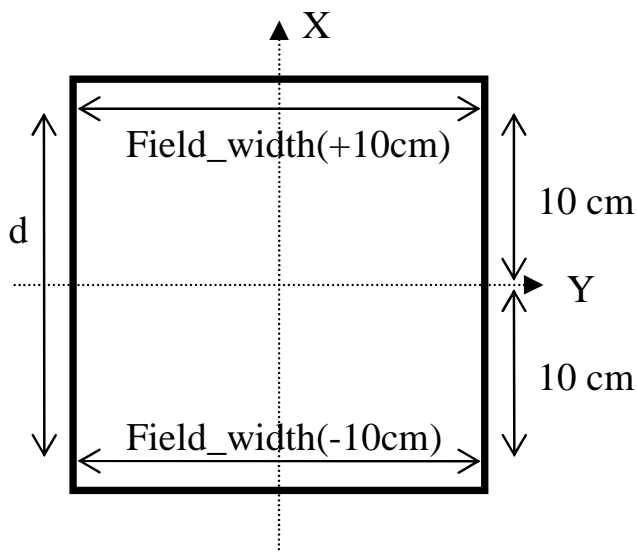


Figure 5: Difference between EPID and film measurements for a prescribed position of $\mathbf{- 5}$ (diamond markers) and $+15 \mathrm{~cm}$ (cross markers). Results are presented without (a-b) and with (cd) the correction for the EPID tilt. Two EPID images were necessary to cover the entire MLC bank length and hence the results are presented separately for the top and the bottom acquisitions.

\section{Without any EPID tilt correction}
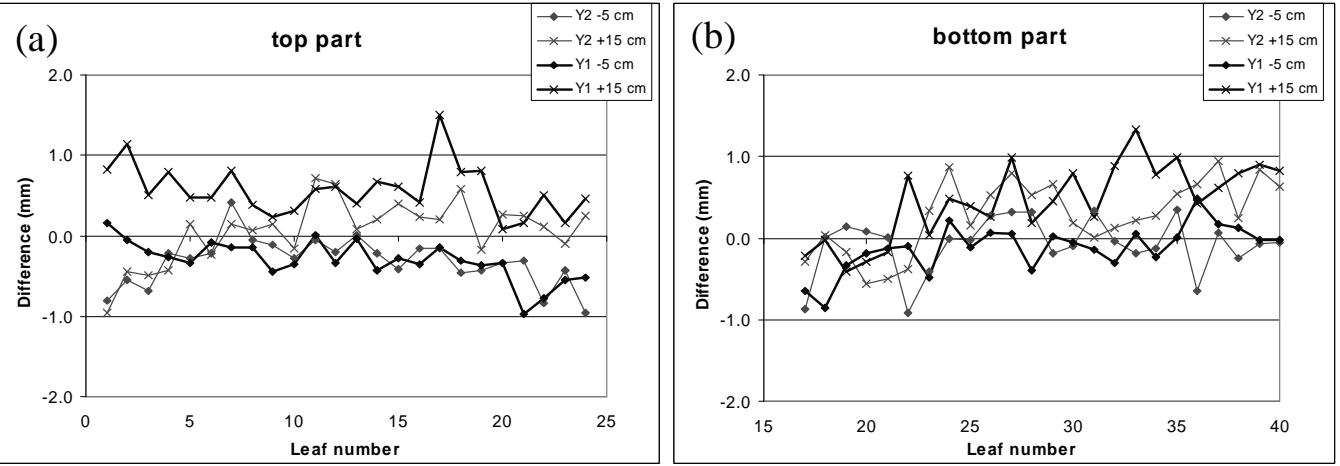

445

With an EPID tilt correction
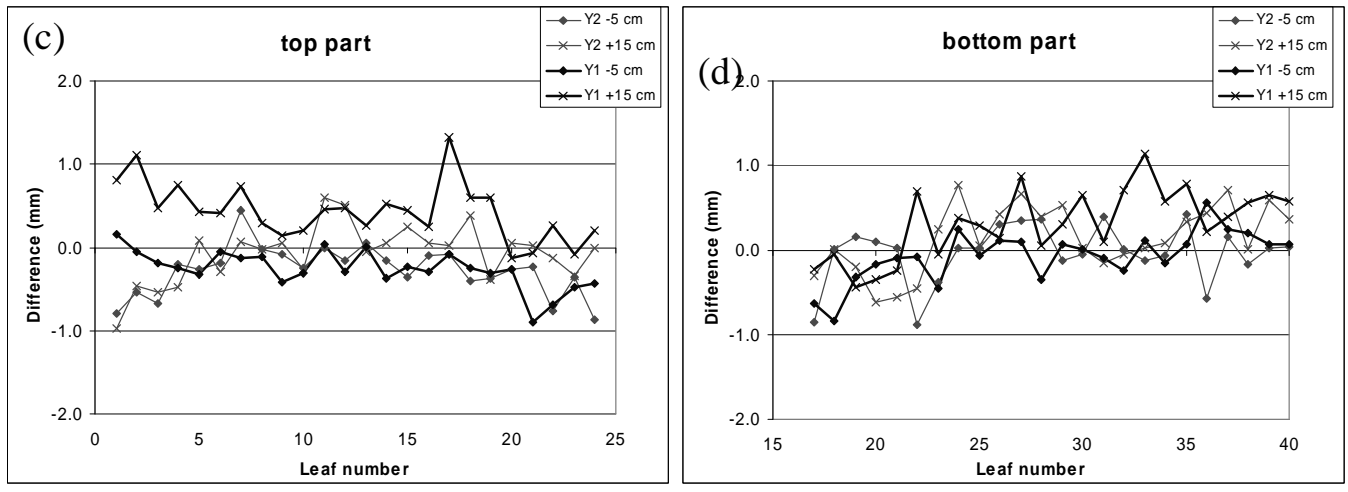
Figure 6: Prescribed, true and predicted positions. a and $b$ represent the difference between the true position and the prescribed position. $c$ and $d$ present the difference between the predicted position using the central leaf $(C L)$ method and the true position. $e$ and $f$ present the difference between the predicted position using the individual leaf (IL) method and the true position. On each graph, each curve represents a prescribed position of the leaf bank $(-11,-5,0,+5,+10$ and $+15 \mathrm{~cm}$ at the isocentre).
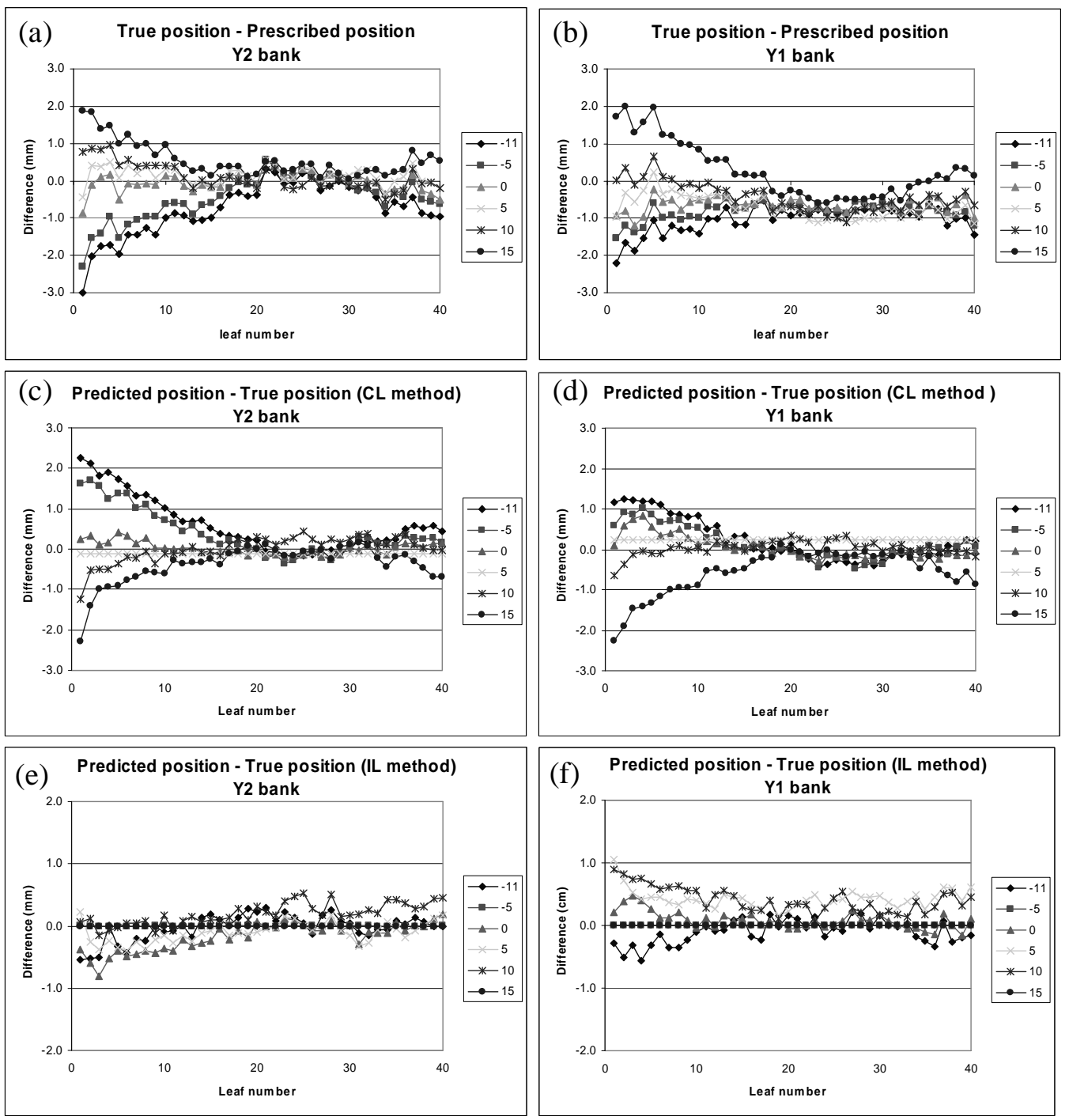
Figure 7: Reproducibility tests. a and b present the difference between the true positions of the leaves measured at an interval of 6 weeks (long term reproducibility). c and d present the difference of the true position measured at 2 gantry angles $\left(90\right.$ and $\left.270^{\circ}\right)$ with the positions measured at $0^{\circ}$. Each curve represents a position of the leaf bank $(-5$ and $+15 \mathrm{~cm}$ at the isocentre).

\section{Long term reproducibility at 6 weeks}
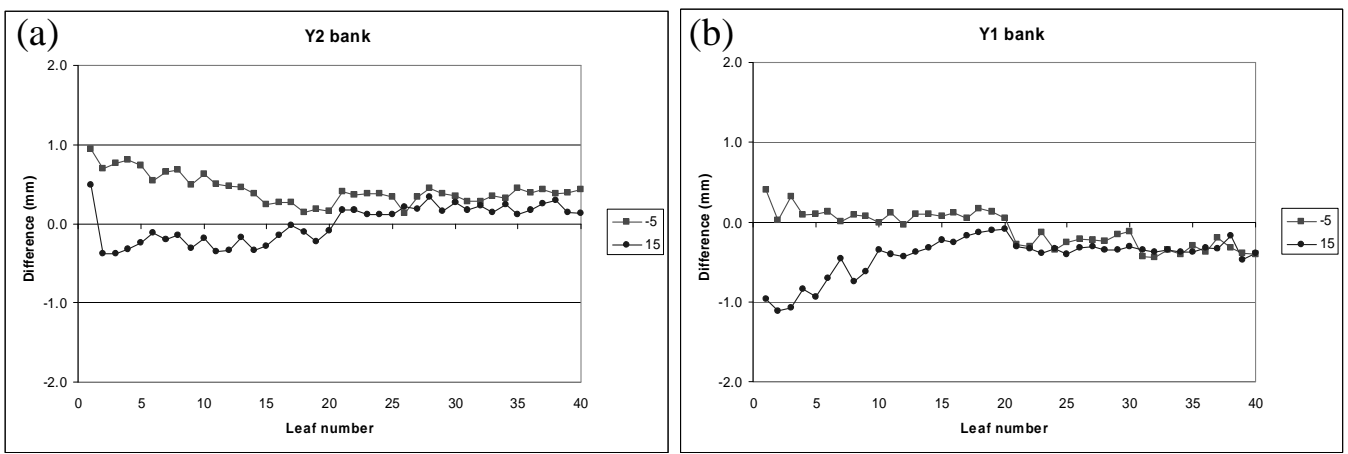

Effect of gravity
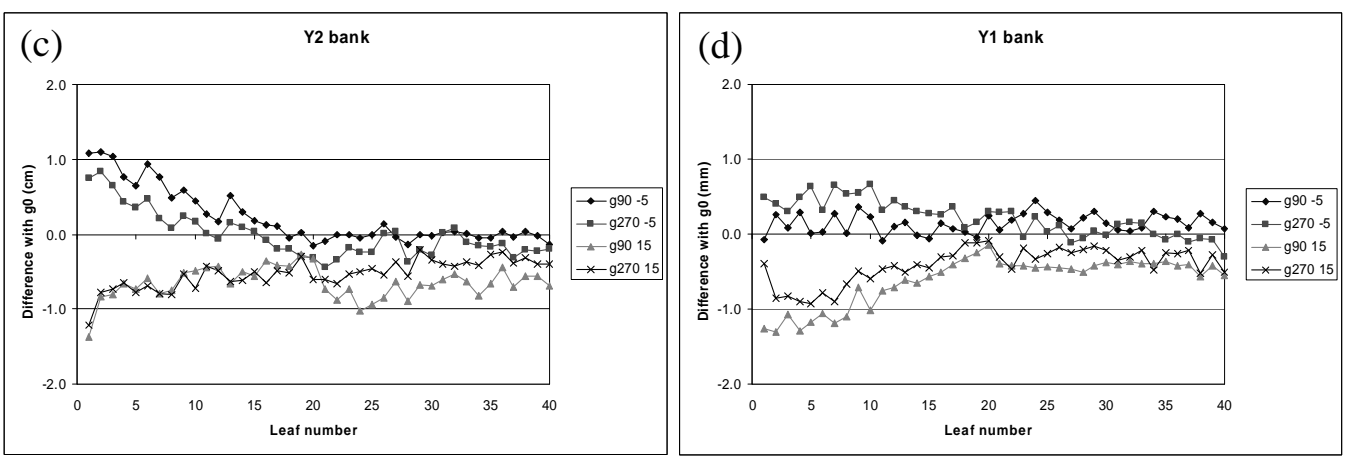
${ }^{1}$ A.R.Hounsell, T.J.Jordan, "Quality control aspects of the Philips multileaf collimator", Radiother. Oncol.45, 225-233 (1997).

${ }^{2}$ M.K.Woo, A.Nico, "Impact of multileaf collimator leaf positioning accuracy on intensity modulation radiation therapy quality insurance ion chamber measurements", Med. Phys.32, 1440-1445 (2005).

${ }^{3}$ International Electrotechnical Commission, "Medical electronical equipment: particular requirements for the safety of electron accelerators in the range $1 \mathrm{MeV}$ to 50 MeV", report No.IEC 60601-2-1(1998).

$485{ }^{4}$ T.J.Jordan, P.C.Williams, "The design and performance characteristics of a multileaf collimator", Phys. Med. Biol.39, 231-251 (1994).

${ }^{5}$ Elekta Oncology systems Ltd, "Precise treatment system. Service mode operators manual", 4513370199952

${ }^{6}$ S.J.Baker, G.J.Budgell, R.I.Mackay, "Use of an amorphous silicon electronic portal

490 imaging device for multileaf collimator quality control and calibration", Phys. Med. Biol.50, 1377-1392 (2005).

${ }^{7}$ W.P.M.Mayles, R.Lake, A.McKenzie, E.M.Macaulay, H.M.Morgan, T.J.Jordan, S.K.Powley, "Physics aspects of quality control in radiotherapy", IPEM(1999). ${ }^{8}$ A.L.Boyer, P.Biggs, J.Galvin, E.Klein, T.LoSasso, D.A.Low, K.Mah, C.X.Yu, 495 "AAPM report No. 72: basic applications of multileaf collimators", (2001).

${ }^{9}$ Varian medical systems, "Millenium MLC systems and maintenance guide", PN 100012956-01

${ }^{10}$ Siemens medical systems, "OPTIFOCUS (82 leaf MLC) Tuneup Component ONCOR/PRIMUS", $7337731 \mathrm{G}$

$500{ }^{11}$ J.M.Lydon, "Theoretical and experimental validation of treatment planning for narrow MLC defined photon fields", Phys. Med. Biol.50, 2701-2714 (2005). 